\title{
Association Between Weight Misperception and Some Mental Health-Related Characteristics in Korean Adolescents
}

This article was published in the following Dove Press journal:

Neuropsychiatric Disease and Treatment

\author{
Keon Hui Lee ${ }^{l, *}$ \\ Su Hyun Bong $\left(\mathbb{D}^{1}\right)^{1, *}$ \\ Dae Hun Kang ${ }^{2}$ \\ Tae Young Choi $\mathbb{B}^{\prime}$ \\ Jun Won Kim (D)
}

'Department of Psychiatry, Catholic University of Daegu School of Medicine, Daegu, Republic of Korea; ${ }^{2}$ Department of Psychiatry, The Armed Forces Daejeon Hospital, Daejeon, Republic of Korea

*These authors contributed equally to this work
Correspondence: Jun Won Kim Catholic University of Daegu School of Medicine, 17-Gil 33 Duryugongwon-Ro, Nam-Gu Daegu 42472, South Korea

Tel $+82-53-650-4780$

Fax+82-53-623-1694

Email f_affection@hotmail.com
Background: Obesity in adolescents is associated with their mental as well as physical health. Adolescents tend to have negative or distorted perceptions about their body weight; however, the effects of such weight misperception on mental health remain unclear. This study investigated the association between weight misperception and mental health in Korean adolescents.

Methods: The analysis was based on the Korea Youth Risk Behavior web-based survey 2017 dataset, which included data from 62,276 middle and high school students at 800 schools throughout Korea. The students were divided into three groups according to whether they overestimated their body weight, underestimated it, or had no misperception. The mental health characteristics of the overestimation and no misperception groups were compared through multiple logistic regression analysis.

Results: Based on their body mass index, male adolescents were more likely to be overweight than female adolescents $(\mathrm{p}<0.001)$ and less likely to have weight misperception. The female adolescents were more likely to overestimate their body weight and less likely to underestimate it $(\mathrm{p}<0.001)$. The male adolescents and female adolescents who overestimated their body weight had significantly lower levels of subjective happiness than their peers had and were more likely to experience subjective stress, sadness/despair, suicidal ideation, and suicide planning. Compared with their peers who have no misperception, male adolescents with overestimation were less likely to drink alcohol (odds ratio [OR], 0.903) or smoke (OR, 0.871), whereas female adolescents with overestimation were more likely to drink alcohol (OR, 1.107) and smoke (OR, 1.130).

Conclusion: Male and female adolescents who overestimated their body weight experienced more psychological problems. Thus, interventions to assist adolescents to gain realistic weight perception may be beneficial. Particularly, more attention needs to be given to female adolescents, who are more likely to overestimate their body weight compared with their male counterparts.

Keywords: weight perception, adolescent, mental health, body mass index, body image

\section{Background}

Obesity rates among Korean adolescents have steadily increased over the recent years, from $8.2 \%$ in 2005 to $8.6 \%$ in 2011 and $13.9 \%$ in $2017 .{ }^{1}$ In approximately $80 \%$ of the cases, obesity in adolescence leads to adult obesity, ${ }^{2}$ and obesity in adolescence is associated with major chronic illnesses and early death in adulthood. $^{3}$ Adolescents with obesity frequently have low self-esteem and a negative self-image ${ }^{4}$ and often develop psychiatric problems, such as somatoform 
disorder, mood disorder, anxiety disorder, dietary disorder, and attention deficit hyperactivity disorder. ${ }^{5-8}$ Other reported consequences of adolescent obesity include missing out on education, decreased family income, increased poverty, and decreased marriage rates. ${ }^{9}$ An increase in the prevalence of obesity among adolescents, thus, affects both adolescent and adult health and is therefore considered an important health issue in many countries. ${ }^{10}$

Because of the widespread social preference for slim physiques, subjective body image and objective measures of body size (such as the body mass index [BMI]) can be the cause of stress to many adolescents. Adolescents are often sensitive to peer evaluation as well as susceptible to influence by the mass media to adopt a negative perception of their weight. ${ }^{11}$ Body image concerns tend to be more prevalent in female adolescents than in their male counterparts, ${ }^{12}$ and most female adolescents in Korea report dissatisfaction with their body weight. ${ }^{13}$ It is also common for adolescents to overestimate their weight even when their weight and body shape are normal. ${ }^{11}$ Discrepancies between their subjective weight perception and their actual weight can cause adolescents unnecessary and excessive stress regarding their weight, which can result in inappropriate weight control behavior as well as other potential psychiatric negative effects such as depression, suicidality, and substance use.

Studies have shown that although physical obesity, determined by objective measures, can be the cause of negative emotions such as depression and anxiety, it is the subjective perception of body shape or weight, rather than body weight itself, that is the more powerful causal factor. ${ }^{14}$ Indeed, weight perception can affect depression and low self-esteem independently of actual body weight. $^{15}$ A negative perception of body weight and shape in adolescence appears to be directly related to psychological problems, such as depression and stress, and additionally increases suicidal ideation and suicide attempts. $^{16}$

Historically, most studies on the relationship between weight perception and mental health have focused on patients with eating disorders ${ }^{17}$ or on specific groups within the general population, such as individuals who are obese or overweight. ${ }^{18}$ Although there have been studies on subjective weight perception and mental health in adolescents, ${ }^{16}$ only a few studies ${ }^{19}$ have examined the relationship between mental health and weight misperception. In one previous study, ${ }^{19}$ perceived weight status had a greater impact on physical self-perceptions and anxiety level than measured weight status for female adolescents. To the best of our knowledge, the present study is one of the largest studies on the relationship between weight misperception and mental health in adolescents. We have expanded on the previous findings in both males and females. Therefore, this study aimed to investigate the relationship between weight misperception and mental health problems, including subjective stress, sadness and despair, suicidality, or substance use, in Korean adolescents.

\section{Methods}

\section{Ethics Statement}

Ethical approval for analysis of publicly available data used in this study was reviewed by the Institutional Review Board of Daegu Catholic University Hospital (DCUMC IRB approval No. CR-20-187) in accordance with the Declaration of Helsinki. The Institutional Review Board of Daegu Catholic University Hospital deemed exempt from ethics review because all data were anonymous.

\section{Population and Classification}

The analysis was based on the Korea Youth Risk Behavior web-based survey conducted in 2017. An online survey of youth health behaviors has been conducted annually since 2005 by the Korea Centers for Disease Control (KCDC). This survey is similar to the National Youth Tobacco Survey and the Youth Risk Behavior Surveillance System organized by the Centers for Disease Control and Prevention (CDC) in the United States. The Korea Youth Risk Behavior questionnaire used in the present study was developed by the KCDC. Its validity and reliability were tested in $2010,{ }^{20}$ revealing that the questionnaire has testretest reliability similar to that of the CDC's Youth Risk Behavior Surveillance System and sufficient validity. The analysis used raw data obtained from the survey's website.

The 2017 survey included national middle and high school students as of April 2017. The sampling for the survey was performed as follows. To minimize sample error, the population was first divided into 117 strata according to 39 regional counties and three school levels (junior, general, and specialized high schools). The number of sample schools was allocated according to the city, province, city size, regional group, whether the schools were boys-only, girls-only, or coeducational, and whether the high schools were general or specialized. Stratified 
colony sampling was used for the sampling, with the primary extraction unit being the school and the secondary extraction unit being the class. The survey was conducted from June 1, 2017 to July 18, 2017. Of the 64,991 potential respondents from the 800 schools, 62,276 students from 799 schools participated in the survey, a participation rate of $95.8 \%$. The present study included data for 60,392 of the respondents, excluding those with missing values for the weight-related variables.

BMI, calculated from the respondent's self-registered height and weight data, was used to determine their actual weight status, with BMI $<18.5 \mathrm{~kg} / \mathrm{m} 2$ considered to be underweight, BMI 18.5-24.9 kg/m2 considered normal, and $\mathrm{BMI}>25 \mathrm{~kg} / \mathrm{m} 2$ considered overweight, in accordance with the World Health Organization Asia-Pacific standard, as applied to Koreans. The respondent's weight perception was assessed with the item, "How do you feel about your body shape?" The response options were "Very Thin," "Not very thin," "Normal," "Not very fat," and "Very fat," with the first two considered to indicate a perception of being underweight and the last two a perception of being overweight. The respondents were then classified into three groups: overestimation (perceived weight status $>$ actual weight status), underestimation (perceived weight status $<$ actual weight status), and normal (perceived weight status corresponds with the actual weight status).

Weight control behaviors were assessed with the question, "Have you tried to control your weight in the last 30 days?" The response options were "Not tried," "Tried to lose weight," "Tried to gain weight," and "Tried to maintain weight."

\section{Measurements}

Sociodemographic variables extracted from the database included age, sex, area of residence, residential type, parental education level, academic achievement, and socioeconomic status. The area of residence was divided into large cities, small cities, and rural areas. Residential type was divided into with the family, with relatives, at a boarding school or residence, and at a care facility. Parental education level was classified as less than middle school graduation, high school graduation, and university graduation. In the survey, academic achievement and socioeconomic status were categorized as "High," "Medium-high," "Medium," "Medium-low," and "Low." For the present study, "High" and "Medium-high" were combined as "High," and "Medium-low" and "Low" were combined as "Low."

Mental health-related characteristics in the analysis included subjective happiness, subjective stress, experience of sadness/despair, history of suicidal ideation, history of suicide planning, history of suicide attempts, history of alcohol consumption, and history of smoking. Subjective happiness was assessed with the question, "How happy do you usually feel?" The response options in the survey were "Very happy," "Somewhat happy," "Moderate," "Somewhat unhappy," and "Very unhappy." However, for the logistic regression analysis, these were categorized into two groups: "Happy," comprising the first two options, or "Moderate/ Unhappy," comprising the last three options. Subjective stress was assessed with the question, "How much stress do you usually feel?" with the response options, "A great deal, "Some," "Not much," "Little," and "None at all." For the Logistic regression analysis, they were categorized into two groups: "Stressed," comprising the first two options, and "Not stressed," comprising the last three options. The experience of sadness/despair was assessed by the question, "Have you been sad or desperate enough to stop your daily life for 2 weeks at any time during the last 12 months?" with the response options "No, not during the past 12 months" and "Yes, I have during the last 12 months." History of suicidal ideation, suicide planning, and suicide attempts was assessed with questions, "Have you seriously considered suicide in the last 12 months?" "Have you made a specific plan to commit suicide in the last 12 months?", and "Have you attempted suicide during the last 12 months?" The response options were "No, not during the last 12 months" or "Yes, during the last 12 months."

\section{Statistical Analysis}

BMI category, weight perception status, and weight control behaviors were compared between the male and female adolescents using a chi-square test. Independent $t$-tests(continuous variables) and chi-square tests(categorical variables) were used to compare the sociodemographic variables between the two weight perception groups (overestimation, normal). Logistic regression analysis helped evaluate the relationships between weight overestimation (as an independent variable) and mental health problems (as the dependent variables) for each sex, calculating odds ratios (ORs) with their 95\% confidence intervals (CIs). The mental health problems considered in the analyses were subjective happiness, subjective stress, experience of sadness/despair, suicidal ideation, suicide planning, suicide attempts, drinking 
alcohol, and smoking. Sociodemographic variables that differed significantly between the two groups (overestimation, normal) were set as covariates, and their effects were statistically adjusted. These included age, parental education level, academic achievement, socioeconomic status, residential type, BMI category, and weight control behavior. Missing data were handled via pairwise deletion. The data were analyzed using IBM SPSS for Windows ver. 18.0 (SPSS Inc., Chicago, IL, USA), and the statistical significance level was set as $\mathrm{p}<0.05$.

\section{Results}

\section{Differences Between the Sexes in Weight and Weight Perception}

The 60,392 respondents included 30,662 male and 29,730 female adolescents. There were no significant differences between the sexes as regards socioeconomic level or residential type. Table 1 compares the BMI, weight perception categories, and weight control behavior between male and female adolescents. There was a significant difference in BMI categorization between the sexes, with a greater proportion of the males classified as overweight. A greater proportion of the male than female adolescents had an accurate perception of their weight category, with a greater proportion of female adolescents overestimating their weight category and significantly fewer underestimating it. Compared with the male adolescents, a much higher proportion of the female adolescents reported trying to lose weight, and very few reported trying to gain weight;

Table I Comparison of Body Weight Characteristics Between the Sexes

\begin{tabular}{|l|l|l|l|}
\hline & $\begin{array}{l}\text { Male } \\
\mathbf{( N = 3 0 , 6 6 2 )}\end{array}$ & $\begin{array}{l}\text { Female } \\
\mathbf{( N = 2 9 , 7 3 0 )}\end{array}$ & $\mathbf{P}$ \\
\hline BMI category & & & \\
Underweight & $6337(20.0 \%)$ & $6890(22.9 \%)$ & $<0.001$ \\
Normal weight & $14,535(47.6 \%)$ & $16,811(57.1 \%)$ & \\
Overweight & $9790(32.4 \%)$ & $6029(20.0 \%)$ & \\
\hline Weight perception & & & \\
Underestimate & $6017(20.3 \%)$ & $1732(5.8 \%)$ & $<0.001$ \\
Normal & $21,011(68.3 \%)$ & $19,015(63.7 \%)$ & \\
Overestimate & $3634(11.4 \%)$ & $8983(30.5 \%)$ & \\
\hline Weight control & & & \\
behavior & & & $<0.001$ \\
None & $16,114(52.7 \%)$ & $12,601(42.4 \%)$ & \\
Tried to lose weight & $7311(23.8 \%)$ & $12,644(42.5 \%)$ & \\
Tried to gain weight & $3709(12.1 \%)$ & $654(2.2 \%)$ & \\
Tried to maintain weight & $3498(11.4 \%)$ & $3831(12.9 \%)$ & \\
\hline
\end{tabular}

as a result, weight control behaviors differed significantly between the sexes $(\mathrm{p}<0.001)$.

\section{Differences in Characteristics Between the Overestimation and Normal Weight Perception Groups}

Tables 2 and 3 compare sociodemographic characteristics and weight control behavior between the overestimation and normal weight perception groups for the male and female adolescents, respectively. For both male and female adolescents, the weight overestimation group was slightly but significantly younger than the normal group. For male adolescents, the overestimation group exhibited significantly better academic achievement than the normal group, whereas the academic achievement of the female overestimation group was significantly lower than that of the normal group. There was no significant difference in the residential area or socioeconomic level between the overestimation and normal groups for either sex. However, there was a significant difference in residence type between the overestimation and normal groups for the male but not the female adolescents. In both sexes, there was a significant difference in weight control behavior between the overestimation and normal groups, with a much higher proportion of the overestimation groups seeking to lose weight.

\section{Weight Overestimation and Mental Health in Male Adolescents}

In the male overestimation group, subjective happiness levels were lower than those of the normal group (OR, 0.830; 95\% CI, 0.765-0.900; $p<0.001$ ) (Table 4). Subjective stress levels (OR, 1.119; 95\% CI, 1.034-1.210; $\mathrm{p}=0.005)$, sadness/despair (OR, 1.136; 95\% CI, 1.037-1.244; $\mathrm{p}=0.006$ ), suicidal ideation (OR, 1.235; 95\% CI, 1.095-1.393; $\mathrm{p}=$ 0.001 ), and suicide planning (OR, 1.235; 95\% CI, 1.095 $1.393 ; \mathrm{p}=0.001)$ were higher in the overestimation group than in the normal group. However, the suicide attempt rate did not differ significantly between the overestimation and normal groups. Alcohol consumption (OR, 0.871; 95\% CI, $0.780-0.971 ; p=0.013)$ and smoking (OR, $0.903 ; 95 \% \mathrm{CI}$, $0.835-0.976 ; p=0.010$ ) were significantly less common in the overestimation group than in the normal group.

\section{Weight Overestimation and Mental Health in Female Adolescents}

In the female overestimation group, subjective happiness levels were significantly lower than those of the normal 
Table 2 Characteristics by Weight Perception Group for the Male Adolescents

\begin{tabular}{|c|c|c|c|c|}
\hline & Normal Group $(\mathrm{N}=2 \mathrm{I}, 0 \mathrm{I} \mid \mathrm{I})$ & Overestimation Group $(\mathbf{N}=3634)$ & $t / \chi^{2}$ & $\mathbf{p}$ \\
\hline Age (years) & 15.07 & 14.27 & 22.024 & $<0.001 *$ \\
\hline \multicolumn{5}{|l|}{ Area of residence } \\
\hline Rural & $1670(6.0 \%)$ & $252(4.7 \%)$ & 9.283 & 0.058 \\
\hline Small city & $10,295(51.1 \%)$ & $1800(51.9 \%)$ & & \\
\hline Large city & 9046 (42.9\%) & 1582 (43.4\%) & & \\
\hline \multicolumn{5}{|l|}{ Paternal education** } \\
\hline College degree or higher & $10,650(52.5 \%)$ & 1793 (52.2\%) & 44.313 & $<0.001 *$ \\
\hline High school education & 5295 (24.7\%) & $792(21.1 \%)$ & & \\
\hline Middle school education or lower & $384(1.7 \%)$ & $54(1.4 \%)$ & & \\
\hline \multicolumn{5}{|l|}{ Maternal education** } \\
\hline College degree or higher & $9776(48.0 \%)$ & $17 \mid 4(49.3 \%)$ & 54.757 & $<0.001 *$ \\
\hline High school education & $6156(29.4 \%)$ & $886(24.3 \%)$ & & \\
\hline Middle school education or lower & $292(1.3 \%)$ & $43(1.0 \%)$ & & \\
\hline \multicolumn{5}{|l|}{ Academic achievement } \\
\hline Low & $6642(31.7 \%)$ & 1074 (29.1\%) & 12.064 & $0.003^{*}$ \\
\hline Middle & $5859(27.9 \%)$ & $1003(27.8 \%)$ & & \\
\hline High & $8150(40.4 \%)$ & 1557 (43.1\%) & & \\
\hline \multicolumn{5}{|l|}{ Socioeconomic status } \\
\hline Low & $2784(13.3 \%)$ & 478 (I2.5\%) & 5.659 & 0.062 \\
\hline Middle & 9178 (43.3\%) & 1525 (42.0\%) & & \\
\hline High & 9049 (43.5\%) & $163 \mid(45.6 \%)$ & & \\
\hline \multicolumn{5}{|l|}{ Residential type } \\
\hline With family & 20,044 (95.8\%) & $3480(96.5 \%)$ & 13.016 & $0.004 *$ \\
\hline With relatives & $170(0.8 \%)$ & $39(1.0 \%)$ & & \\
\hline With friends, alone, dormitory & $728(3.1 \%)$ & $96(2.1 \%)$ & & \\
\hline Care facility & $69(0.3 \%)$ & $19(0.4 \%)$ & & \\
\hline \multicolumn{5}{|l|}{ Weight control behavior } \\
\hline None & 10,825 (51.9\%) & 1859 (51.0\%) & 180.328 & $<0.001 *$ \\
\hline Tried to lose weight & $5428(25.8 \%)$ & $1088(30.2 \%)$ & & \\
\hline Tried to gain weight & $2347(11.0 \%)$ & $|8|(4.7 \%)$ & & \\
\hline Tried to maintain weight & $24 I I(11.3 \%)$ & $506(14.0 \%)$ & & \\
\hline
\end{tabular}

Notes: ${ }^{*} \mathrm{p}<0.05$; **Paternal and maternal education have missing values because of the students who do not know their parents' education level.

group (OR, 0.783; 95\% CI, 0.747-0.846; p < 0.001). Subjective stress levels (OR, 1.339; 95\% CI, 1.262-1.420; $\mathrm{p}<0.001$ ), sadness/despair (OR, 1.136; 95\% CI, 1.037-1.244; $\mathrm{p}=0.006)$, suicidal ideation (OR, 1.203; 95\% CI, 1.233 $1.291 ; \mathrm{p}<0.001)$, and suicide planning (OR, 1.188; 95\% CI, $1.049-1.344)$ were significantly high in the overestimation group than in the normal group. The suicide attempt rate did not differ significantly between the two groups. Unlike the male adolescents, alcohol consumption (OR, 1.107; 95\% CI, $1.046-0.976 ; \mathrm{p}=0.010)$ and smoking (OR, $0.130 ; 95 \% \mathrm{CI}$, $1.015-1.258 ; \mathrm{p}=0.025)$ were significantly more common among the female adolescents in the overestimation group compared with those in the normal group.

\section{Discussion}

This study analyzed the relationship between weight misperception and mental health using data extracted from the 2017 Korean Youth Risk Behaviors online survey, which collected data from 62,276 male and female students enrolled in the first grade of a junior high school to the third grade of a high school. Although there have been previous studies on mental health and body weight, including subjective perceptions of body weight, most focused on subjective weight perceptions without considering BMI. We hypothesized that health behaviors may differ between those who were categorized as overweight as per their BMI and those who were 
Table 3 Characteristics by Weight Perception Group for the Female Adolescents

\begin{tabular}{|c|c|c|c|c|}
\hline & Normal Group $(N=19,015)$ & Overestimation Group $(\mathbf{N}=\mathbf{8 9 8 3})$ & $t / \chi 2$ & $\mathbf{p}$ \\
\hline Age (years) & 15.18 & 14.90 & 11.182 & $<0.001 *$ \\
\hline \multicolumn{5}{|l|}{ Area of residence } \\
\hline Rural & 1456 (6.4\%) & $624(5.8 \%)$ & 4.013 & 0.174 \\
\hline Small city & 8852 (50.2\%) & $4236(51.0 \%)$ & & \\
\hline Large city & 8707 (43.4\%) & $4123(43.2 \%)$ & & \\
\hline \multicolumn{5}{|l|}{ Paternal education** } \\
\hline College degree or higher & 9671 (52.5\%) & $4658(53.4 \%)$ & 40.973 & $<0.001 *$ \\
\hline High school education & $5043(26.4 \%)$ & $2248(24.7 \%)$ & & \\
\hline Middle school education or lower & $398(2.0 \%)$ & $118(1.2 \%)$ & & \\
\hline \multicolumn{5}{|l|}{ Maternal education** } \\
\hline College degree or higher & $8915(47.9 \%)$ & $4298(48.9 \%)$ & 21.256 & $<0.001 *$ \\
\hline High school education & 6437 (34.3\%) & $2900(32.3 \%)$ & & \\
\hline Middle school education or lower & $356(1.8 \%)$ & $139(1.4 \%)$ & & \\
\hline \multicolumn{5}{|l|}{ Academic achievement } \\
\hline Low & $5991(31.8 \%)$ & 2995 (33.7\%) & 9.939 & $0.009 *$ \\
\hline Middle & $5762(30.6 \%)$ & 2667 (29.7\%) & & \\
\hline High & $7262(37.6 \%)$ & $3321(36.6 \%)$ & & \\
\hline \multicolumn{5}{|l|}{ Socioeconomic status } \\
\hline Low & $2715(13.9 \%)$ & $1323(14.4 \%)$ & 2.828 & 0.270 \\
\hline Middle & $9308(48.8 \%)$ & $4406(49.2 \%)$ & & \\
\hline High & $6992(37.3 \%)$ & $3200(36.3 \%)$ & & \\
\hline \multicolumn{5}{|l|}{ Residential type } \\
\hline With family & 18,152 (95.9\%) & $8622(96.4 \%)$ & 6.019 & 0.082 \\
\hline With relatives & $100(0.5 \%)$ & 43 (0.4\%) & & \\
\hline With friends, alone, dormitory & $698(3.3 \%)$ & $298(3.0 \%)$ & & \\
\hline Care facility & $65(0.3 \%)$ & $20(0.2 \%)$ & & \\
\hline \multicolumn{5}{|l|}{ Weight control behavior } \\
\hline None & 8321 (44.4\%) & 3431 (38.2\%) & 403.166 & $<0.001 *$ \\
\hline Tried to lose weight & $7675(40.4 \%)$ & 4367 (48.8\%) & & \\
\hline Tried to gain weight & $576(3.0 \%)$ & $43(0.4 \%)$ & & \\
\hline Tried to maintain weight & $2443(12.6 \%)$ & 1142 (12.6\%) & & \\
\hline
\end{tabular}

Notes: ${ }^{*} \mathrm{p}<0.05$; **Paternal and maternal education have missing values because of the students who do not know their parents' education level.

underweight or normal weight. Therefore, this study explored the association between mental health factors and weight misperception, taking into account both BMI and subjective weight perception.

Previous studies have reported differences in health behavior between male and female subjects. Fewer women than men are obese, yet women are more likely to perceive themselves as obese (relative to their actual weight) and exhibit weight control behavior. ${ }^{21,22}$ Because of this, we analyzed the weight perception and weight control behavior data for male and female adolescents separately.
More female than male adolescents overestimated their weight, whereas more male adolescents underestimated their body weight. One reason for this difference may be the differing criteria for the "ideal" body shape for men and women. ${ }^{23}$ During socialization, adolescents are influenced by various sociocultural factors projected by their peers and the mass media. For men and male adolescents, a muscular body type is considered ideal, whereas many women and female adolescents consider a slim body type to be ideal or even standard. However, these notions are, in fact, unrealistic, and maladaptive from the perspective of general health. Nevertheless, adolescents often cultivate 


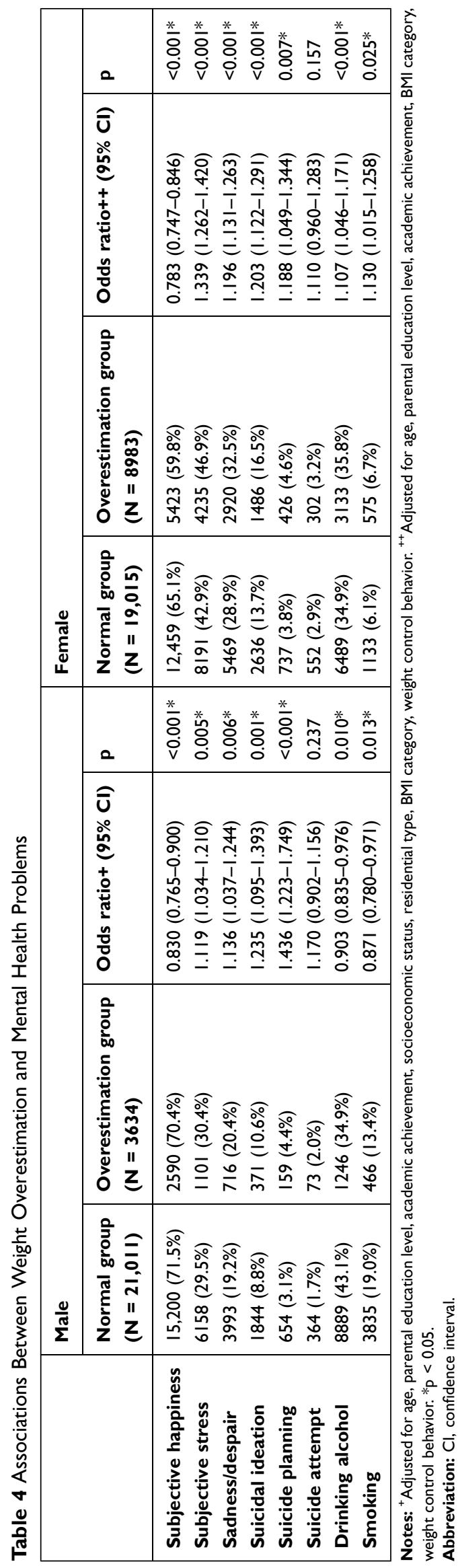

attitudes based on these "ideals," with male adolescents tending to perceive themselves as thin and female adolescents tending to perceive themselves as fat. This finding is consistent with studies which found that male adolescents who perceived themselves as "underweight" exhibited lower levels of body satisfaction and self-esteem, whereas female adolescents who perceived themselves as "underweight" had higher levels of body satisfaction and selfesteem ${ }^{24}$ again, this suggested that female adolescents want to be thinner and male adolescents want to be larger. $^{25}$

Among those who overestimated their body weight in both sexes, subjective happiness was lower, and subjective stress, sadness/despair, suicidal ideation, and suicide planning were higher, compared with those who have a normal estimation of their body weight. Studies have shown that individuals with high BMI commonly experience weightrelated stigma and discrimination in a variety of daily situations, including at school and work ${ }^{26}$ and that this stigma is associated with negative mental consequences, such as body dissatisfaction, depression, and eating disorders. ${ }^{26,27}$ It is possible that weight-related stigmatization or bullying that starts in childhood can result in feelings of stress and sadness/despair. Furthermore, studies have reported that adolescents who overestimate their weight have a higher risk of negative mental consequences, such as weight dissatisfaction, ${ }^{28}$ low selfesteem, ${ }^{29}$ and depression. ${ }^{16,30}$ These factors are associated with suicidal ideation in adolescents; ${ }^{31}$ hence, this finding may help explain why a misperception of being overweight is associated with suicidal ideation and suicide planning.

A study on weight perception reported that alcohol consumption and smoking were greater in both male and female adolescents who perceived themselves as overweight. ${ }^{32}$ In the present study, self-reported rates of drinking and smoking were higher among female adolescents who perceived themselves as overweight compared with their male counterparts. The high smoking rate observed in the female overestimation group might be associated with smoking for weight loss, based on the belief that smoking reduces weight. ${ }^{33}$ Adolescents who perceive themselves as overweight might drink alcohol to cope with the negative emotions that result from such perceptions. ${ }^{34}$ Given that the male adolescents who overestimated their weight tended to consume less alcohol, it is possible that they changed their drinking behaviors to prevent additional weight gain. ${ }^{35}$ Studies have indicated 
that male adolescents are less likely to use tobacco for weight control than female adolescents, ${ }^{36}$ which may partially account for the observed difference in smoking rates between male and female adolescent groups. Our study had several strengths, including its large sample size and that it considered body weight misperception rather than only body weight or body weight perception. However, it had several limitations. First, we used questionnaire responses rather than objectively measured data to calculate BMI. Future studies should use BMI data obtained from objective, measured values to evaluate weight misperceptions. Second, each mental health variable was based on a single symptom-based question rather than the scale that is typically used to assess that variable in clinical practice. Future research will benefit from the use of clinical scales that can more clearly and accurately assess mental health, including subjective happiness, stress, and depression, among adolescents. Third, excluding data from students who died secondary to suicide might affect the association between suicidal attempt and mental health problems.

The results of this study showed that, among adolescents, weight misperception is an important mental health consideration. Accurate weight awareness is necessary for maintaining optimal mental health, and there is a need for strategies to address misperceptions among students who incorrectly perceive their weight as deviating from normal standards. Counseling and education are necessary to help adolescents recognize healthy and appropriate body shapes and to establish a healthy body image. Moreover, efforts across society are needed to change the standard or "ideal" body shape to one that is attainable and, more importantly, healthy; this need appears to be especially urgent for female adolescents.

\section{Conclusions}

This study found that more male Korean adolescents were overweight compared with their female counterparts as determined by their BMI; yet, weight misperception and weight control behaviors were higher among female adolescents. Adolescents who overestimated their weight had lower subjective happiness and more psychological problems, including stress, feelings of sadness/ despair, suicidal ideation, and suicide planning. Therefore, interventions are needed for adolescents, especially female adolescents, with weight misperceptions. The results of this study may help prevent and manage mental health problems in adolescents with weight misperceptions, thereby potentially enhancing their overall mental health during the formative adolescent years. Future research in this field should use standard clinical measurements to improve upon our results.

\section{Abbreviations}

BMI, body mass index; ADHD, attention deficit hyperactivity disorder; OR, odds ratio; CI, confidence interval; KCDC, Korea Centers for Disease Control.

\section{Data Sharing Statement}

The datasets used and/or analyzed during the current study are available from the corresponding author on reasonable request.

\section{Consent for Publication}

Not applicable

\section{Acknowledgments}

The English language used in this document has been checked by a professional editor, a native English speaker.

\section{Author Contributions}

All authors made a significant contribution to the work reported, whether that is in the conception, study design, execution, acquisition of data, analysis and interpretation, or in all these areas; took part in drafting, revising or critically reviewing the article; gave final approval of the version to be published; have agreed on the journal to which the article has been submitted; and agree to be accountable for all aspects of the work.

\section{Funding}

There is no funding to report.

\section{Disclosure}

The authors report no conflicts of interest in this work.

\section{References}

1. Healthwise knowledgebase homepage on the internet. Seoul: Korea National Statistical Office; 2017. Available from: http://www.index.go. kr. Accessed September 15, 2019. Korean.

2. Simmonds M, Llewellyn A, Owen CG, Woolacott N. Predicting adult obesity from childhood obesity: a systematic review and meta-analysis. Obes Rev. 2016;17(2):95-107. doi:10.1111/obr.12334

3. Reilly JJ, Kelly J. Long-term impact of overweight and obesity in childhood and adolescence on morbidity and premature mortality in adulthood: systematic review. Int $J$ Obes. 2011;35(7):891-898. doi:10.1038/ijo.2010.222 
4. Sahoo K, Sahoo B, Choudhury AK, Sofi NY, Kumar R, Bhadoria AS. Childhood obesity: causes and consequences. J Family Med Prim Care. 2015;4(2):187-192. doi:10.4103/2249-4863.154628

5. Puder JJ, Munsch S. Psychological correlates of childhood obesity. Int J Obes. 2010;34(Suppl 2):S37-S43. doi:10.1038/ijo.2010.238

6. Pulgaron ER. Childhood obesity: a review of increased risk for physical and psychological comorbidities. Clin Ther. 2013;35(1): A18-A32. doi:10.1016/j.clinthera.2012.12.014

7. Kalarchian MA, Marcus MD. Psychiatric comorbidity of childhood obesity. Int Rev Psychiatry. 2012;24(3):241-246. doi:10.3109/ 09540261.2012.678818

8. Britz B, Siegfried W, Ziegler A, et al. Rates of psychiatric disorders in a clinical study group of adolescents with extreme obesity and in obese adolescents ascertained via a population based study. Int J Obes Relat Metab Disord. 2000;24(12):1707-1714. doi:10. 1038/sj.ijo.0801449

9. Gortmaker SL, Must A, Perrin JM, Sobol AM, Dietz WH. Social and economic consequences of overweight in adolescence and young adulthood. $N$ Engl J Med. 1993;329(14):1008-1012. doi:10.1056/ NEJM199309303291406

10. Ebbeling CB, Pawlak DB, Ludwig DS. Childhood obesity: public-health crisis, common sense cure. Lancet. 2002;360 (9331):473-482. doi:10.1016/S0140-6736(02)09678-2

11. Cho JH, Han SN, Kim JH, Lee HM. Body image distortion in fifth and sixth grade students may lead to stress, depression, and undesirable dieting behavior. Nutr Res Pract. 2012;6(2):175-181. doi:10.41 62/nrp.2012.6.2.175

12. Chung IJ, Lee JY. The impact of obesity and satisfaction with appearance on self-esteem in adolescence focusing on the moderating effect of gender. Mental Health Social Work. 2011;38(8):60-80.

13. Lee WO, Hwang YY. A study on high-school female adolescents' obesity, attitudes towards body and weight control. Youth Facility Environment. 2009;7(3):3-11.

14. Atlantis E, Ball K. Association between weight perception and psychological distress. Int J Obes. 2008;32(4):715-721. doi:10.1038/sj. ijo.0803762

15. Ali MM, Fang H, Rizzo JA. Body weight, self-perception and mental health outcomes among adolescents. J Ment Health Policy Econ. 2010;13(2):53-63.

16. Hwang IC, Lee KS, Park DK, et al. [Association with self-perception for obesity and mental health among Korean adolescent]. J Korean Acad Child Adolesc Psychiatry. 2011;22(2):112-119. Korean. doi:10.5765/JKACAP.2011.22.2.112

17. Hwang RH. [A study on eating disorder, body Image and self-esteem of female university students]. Korean $J$ Women Health Nurs. 2009;15(4):328-335. Korean. doi:10.4069/kjwhn.2009.15.4.328.

18. Nam SJ, Park JH. [Depression and stress related to obesity among normal, obese, and severe obese groups, comparison among normal, obesity, and severe obesity groups]. Korean J Human Ecol. 2012;21 (6):1199-1210. Korean. doi:10.5934/KJHE.2012.21.6.1199.

19. Dolenc P. Relationships between actual and perceived body weight, physical self-concept and anxiety among adolescent girls. JPER. 2019;27(1):25-45.

20. Bae J, Joung H, Kim JY, Kwon KN, Kim YT, Park SW. Test-retest reliability of a questionnaire for the korea youth risk behavior web-based survey. J Prev Med Public Health. 2010;43(5):403-410. doi:10.3961/jpmph.2010.43.5.403
21. Oh DN, Kim EM, Kim SH. [Weight control behaviors and correlates in Korean adolescents]. J Korea Contents Association. 2013;13 (3):218-228. Korean. doi:10.5392/JKCA.2013.13.03.218.

22. Kang HJ. Factors influencing Korean adolescents' body weight perceptions and weight change efforts. Perspectives Nursing Sci. 2012;9 (1):24-35.

23. Flament MF, Hill EM, Buchholz A, Henderson K, Tasca GA, Goldfield G. Internalization of the thin and muscular body ideal and disordered eating in adolescence: the mediation effects of body esteem. Body Image. 2012;9(1):68-75. doi:10.1016/j.bodyim.2011. 07.007

24. Nam SJ, Park JH. Adolescents' satisfaction of body and self-esteem according to obesity and subjective perception of body: verification of moderating effect of sex. Korean J Obes. 2013;22(4):251-253. doi:10.7570/kjo.2013.22.4.251.

25. Cash TF. Cognitive-behavioral perspectives on body image. In: Cash TF, Smolak L, editors. Body Image: A Handbook of Science, Practice, and Prevention. New York: The Guilford Press; 2011:39-47.

26. Puhl RM, Heuer CA. Obesity stigma: important considerations for public health. Am J Public Health. 2010;100(6):1019-1028. doi:10.2105/AJPH.2009.159491

27. Kim Y, Austin SB, Subramanian SV, Kawachi I. Body weight perception, disordered weight control behaviors, and depressive symptoms among Korean adults: the Korea national health and nutrition examination survey 2014. PLoS One. 2018;13(6):e0198841. doi:10.1371/journal.pone.0198841

28. Page RM, Allen O. Adolescent perceptions of body weight and weight satisfaction. Percept Mot Skills. 1995;81(1):81-82. doi:10.24 66/pms.1995.81.1.81

29. Dietz WH. Health consequences of obesity in youth: childhood predictors of adult disease. Pediatrics. 1998;101(Suppl2):518-525.

30. Oh JW, Kim YS, Kwon HJ, Kim DH. Relationship between body weight perception and mental health in Korean adolescents. Korean J Physical Education. 2016;55(1):247-260.

31. Stein D, Orbach I, Shani-Sela M, et al. Suicidal tendencies and body image and experience in anorexia nervosa and suicidal female adolescent inpatients. Psychother Psychosom. 2003;72(1):16-25. doi:10.1159/000067183

32. Lim H, Wang Y. Body weight misperception patterns and their association with health-related factors among adolescents in South Korea. Obesity. 2013;21(12):2596-2603. doi:10.1002/oby.20361

33. Stice E, Shaw H. Prospective relations of body image, eating, and affective disturbances to smoking onset in adolescent girls: how Virginia slims. J Consult Clin Psychol. 2003;71(1):129-135. doi:10.1037//0022-006x.71.1.129

34. Kuntsche E, Knibbe R, Gmel G, Engels R. Who drinks and why? A review of socio-demographic, personality, and contextual issues behind the drinking motives in young people. Addict Behav. 2006;31 (10):1844-1857. doi:10.1016/j.addbeh.2005.12.028

35. Ramseyer Winter V, Kennedy AK, O’Neill E. Adolescent tobacco and alcohol use: the influence of body image. J Child Adolesc Subst Abuse. 2017;26(3):219-228. doi:10.1080/1067828X.2017.1279992

36. Dowdell EB, Santucci ME. Health risk behavior assessment: nutrition, weight, and tobacco use in one urban seventh-grade class. Public Health Nurs. 2004;21(2):128-136. doi:10.1111/j.0737-1209. 2004.021206.x 


\section{Publish your work in this journal}

Neuropsychiatric Disease and Treatment is an international, peerreviewed journal of clinical therapeutics and pharmacology focusing on concise rapid reporting of clinical or pre-clinical studies on a range of neuropsychiatric and neurological disorders. This journal is indexed on PubMed Central, the 'PsycINFO' database and CAS, and is the official journal of The International Neuropsychiatric Association (INA). The manuscript management system is completely online and includes a very quick and fair peer-review system, which is all easy to use. Visit http://www.dovepress.com/testimonials.php to read real quotes from published authors.

Submit your manuscript here: https://www.dovepress.com/neuropsychiatric-disease-and-treatment-journal 